\title{
Towards the development of a wearable temperature sensor based on a ferroelectric capacitor
}

\author{
Stuart Hannah, Helena Gleskova \\ Department of Electronic and Electrical Engineering \\ University of Strathclyde \\ Glasgow, G1 1XW, United Kingdom \\ stuart.hannah@strath.ac.uk, helena.gleskova@strath.ac.uk
}

\author{
Slavomir Matuska, Robert Hudec \\ Department of Multimedia and Information-Communication \\ Technology \\ University of Žilina \\ 01026 Žilina, Slovakia
}

slavomir.matuska@fel.uniza.sk,robert.hude@fel.uniza.sk

\begin{abstract}
Response of a ferroelectric capacitor to static temperature $\left(\sim 22\right.$ to $\left.90^{\circ} \mathrm{C}\right)$ is presented. The sensor is based on ferroelectric ceramic lead zirconate titanate (PZT). The PZT sensor is a cheap, commercially available element used to provide a proof of concept for the initial investigation into using ferroelectric materials for the monitoring of static temperature. The capacitor response to temperature was measured using PZT capacitance changes recorded at $1 \mathrm{kHz}$. The capacitance was measured after the temperature had stabilised. We have found that the PZT capacitor responds linearly as a function of applied temperature, with a sensitivity $\sim 53 \mathrm{pF} /{ }^{\circ} \mathrm{C}$. Furthermore, to provide some initial electronics capable of measuring sensor capacitance in real time, the PZT element was attached to an Arduino Uno platform. Again, the sensor continues to respond linearly to temperature with a sensitivity of $146 \mathrm{pF} /{ }^{\circ} \mathrm{C}$. The system developed paves the way for further work to be done on using ferroelectric materials for the monitoring of static temperature changes, for applications such as human body temperature measurement using a textile-based smart-shirt setup.
\end{abstract}

Keywords - PZT, Capacitance, Temperature, , Electronics, Ferroelectric, Wearables.

\section{INTRODUCTION}

Over the last few years, one area of increasing interest which is rapidly becoming a hot topic is wearable electronics, which is projected to become worth hundreds of billions of dollars annually within the next decade [1]. Wearable electronics has the potential to open up new opportunities to monitor various aspects of human health, such as body temperature, using non-invasive solutions such as e-textiles [2] and smart sensor systems [3]. For example, within the automotive industry, the monitoring of HGV or train drivers' health for safety and personal reasons could employ the use of wearable electronics in the form of sensors. Examples include electrocardiogram (ECG) sensors [4] and pulse sensors [5] etc. for the measurement of vital signs.

Previously, various types of sensor have been shown to monitor temperature using different mechanisms. Examples include resistance temperature detector (RTD) sensors [6], pyroelectric sensors [7, 8] and capacitive sensors [9].
This paper presents the use of a commercially available, cheap ferroelectric sensor based on lead zirconate titanate (PZT) applied to a developing wearable electronics setting, whereby temperature of everyday objects including human body temperature can be measured continuously. Such sensors could be integrated into so-called "smart shirts" for real-time and cost-effective body temperature sensing.

Due to the ferroelectric nature of PZT and its subsequent fabrication process involving steps such as poling, the PZT disc is capable of responding to external stimuli in different ways. Dynamic changes in pressure can be monitored using its piezoelectric effect and dynamic temperature gradients can be monitored through pyroelectricity. To provide a route for monitoring slow changes in temperature without the need for additional circuitry such as a charge amplifier to harness the charge developed through pyroelectricity, the PZT sensor was investigated through changes in PZT capacitance as a function of static temperature. The capacitance of the PZT sensor is able to remain constant, until a new temperature is set. This eliminates the need for a charge amplifier circuit with a long time constant to maintain the set level. Using PZT capacitance changes, steady-state temperature can be monitored easily and effectively.

As a proof of concept, the PZT sensors were subsequently attached to an Arduino Uno and code was developed to measure PZT capacitance in real-time at different sensor temperatures. This paper investigates the capacitive response of the PZT ferroelectric capacitor to temperature to allow the exploitation of ferroelectric devices for static temperature monitoring.

This paper is organized as follows: Section II presents experimental details on the PZT capacitor and describes the measurement procedures used. Results of the sensor response to changing temperature are provided in section III. Finally, discussion is provided and conclusions are drawn in sections $\mathrm{IV}$ and $\mathrm{V}$ respectively.

\section{EXPERIMENTAL SECTION}

\section{A. Selecting a Template (Heading 2)}

Cheap, commercially available ferroelectric lead zirconate titanate (PZT) elements (Murata) were used to develop a proof 
of concept and establish a primary route for cheap and robust temperature monitoring. The circular PZT element consisted of a $0.23 \mathrm{~mm}$-thick PZT film, and measured $20 \mathrm{~mm}$ in diameter. The PZT disc is sandwiched between a brass bottom electrode $(\mathrm{d}=0.3 \mathrm{~mm})$ and a silver $(\mathrm{Ag})$ top electrode $(\mathrm{d} \sim \mathrm{nm}$ 's $)$ in a parallel plate structure. Short wires $(\sim \mathrm{cm}$ 's) were soldered to each electrode for compatibility with the measurement equipment. The circular sensor has an active sensing area of $3.14 \mathrm{~cm}^{\wedge} 2$. At room temperature under normal conditions, the PZT sensor has a nominal capacitance of $~ 10 \mathrm{nF}$. Fig.1 (a) shows a photograph of the ferroelectric PZT sensor and (b) the cross-section of the PZT sensor showing the PZT sandwiched between brass and Ag electrodes in a parallel plate structure.

\section{B. Capacitance of PZT Sensor Versus Temperature}

PZT sensor capacitance was measured as a function of static temperature between $\sim 22$ and $90^{\circ} \mathrm{C}$. Upon application of temperature to the PZT element, the capacitance of PZT changes as a result of a change in the relative permittivity $\varepsilon_{-} r$ of PZT. Since temperature is the only external stimuli of interest, PZT capacitance is not affected by changes in sensor area or thickness.

Sensor capacitance was measured after the temperature had stabilised for at least 30 seconds. Capacitance measurements were performed using an R\&S HM8118 LCR meter. The LCR meter is capable of measuring at frequencies between $20 \mathrm{~Hz}$ and $200 \mathrm{kHz}$, with a resolution of $10 \mathrm{fF}$. In all cases, capacitance was extracted at $1 \mathrm{kHz}$ using an a.c oscillation level of $100 \mathrm{mV}$ with $0 \mathrm{~V}$ d.c bias.
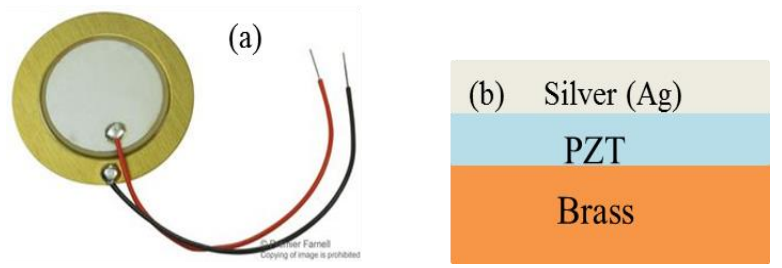

Fig. 1. (a) Ferroelectric PZT sensor where PZT is sandwiched between brass and $\mathrm{Ag} \mathrm{[10].} \mathrm{(b)} \mathrm{The} \mathrm{cross-sectional} \mathrm{view} \mathrm{of} \mathrm{the} \mathrm{sensor.}$

Temperature was applied to the ferroelectric sensor using a digital hotplate (Weller WHP 1000), and recorded in real-time using an infrared thermal imaging camera (FLIR). For several temperatures between room temperature $\left(\sim 22^{\circ} \mathrm{C}\right)$ and $90^{\circ} \mathrm{C}$, PZT capacitance at $1 \mathrm{kHz}$ was extracted.

\section{Capacitance of PZT Sensor Using Ardunio Uno}

As a first step towards developing a complete wearable electronics solution for the measurement of temperature, the PZT sensor was connected to an Uno (Arduino) platform via a $\sim 470 \mathrm{nF}$ capacitor connected in parallel. The role of this capacitor was to reduce the undesired piezoelectric charges developed during the unintentional bending of the PZT sensor. The chosen capacitor also reduced any pyroelectric charge associated with changing temperature that would complicate the temperature calibration of the sensor. PTZ sensor is connected to the Uno via two pins, A0 and A2. A0 serves as charging pin and A2 as discharging. To measure the capacitance, we are relying on basic capacitor property - the time constant TC. The time constant of a capacitor is defined as the time it takes for the voltage across the capacitor to reach $63.2 \%$ of its voltage when fully charged. From the formula for calculation of the voltage across capacitor (1), we can derive the final formula for the PTZ capacitance calculation (2).

$$
\begin{aligned}
& V_{C}=V_{S}\left(1-e^{-\frac{t}{R C}}\right) \\
& C=-\frac{t}{R * \ln \left(1-\frac{V_{C}}{V_{S}}\right)}
\end{aligned}
$$

where $C$ is capacitance, $t$ is sensor charge time when rich $50 \%$ of its maximum possible voltage, $R$ - value of internal Arduino pull-up resistor, $V_{c}$ is measured voltage on sensor and $V_{s}$ is maximal voltage. Code was developed to enable the Uno microcontroller to measure PZT capacitance continuously and output a mean value of 10 readings every 5 seconds. The block diagram is shown in Fig. 2.

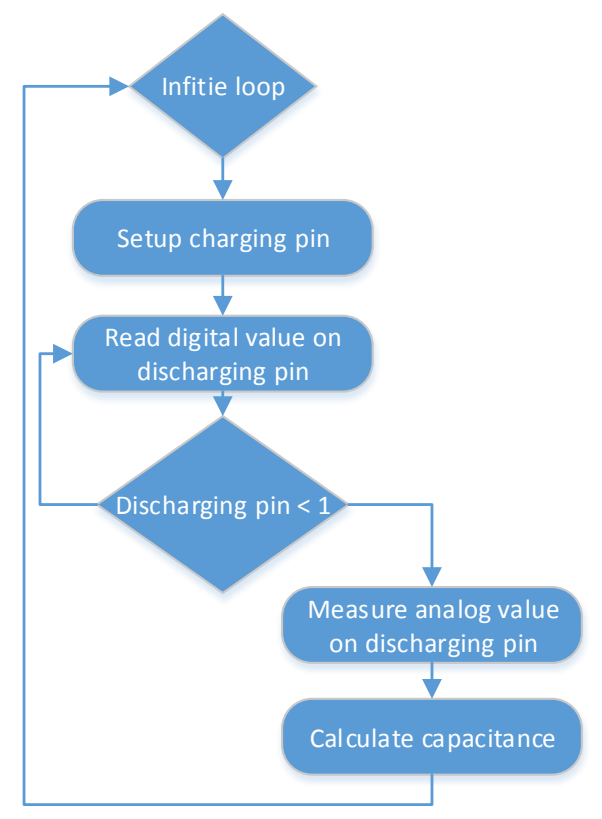

Fig. 2. Block diagram of measurement system

If faster changes in temperature were to occur, the code could be adapted to take readings more often and output data more frequently if necessary. This sensor platform was tested between 36 and $50^{\circ} \mathrm{C}$ to provide information in a temperature range more akin to that of the human body. The measurement resolution is $0.5^{\circ} \mathrm{C}$. The resolution is dependent on the analog to digital convertor (A/D) resolution and on the speed of Arduino microprocessor. For the time measurement, Arduino function micro() was used. Its resolution is only $4 \mu \mathrm{s}$ and A/D resolution is $2^{10}$. For much more accurate resolution, we can use the microcontroller with more precise time measurement and more accurate $\mathrm{A} / \mathrm{D}$ convertor.

\section{RESULTS}

\section{A. Response of PZT to Temperature}

Fig. 3 presents PZT sensor capacitance extracted at $1 \mathrm{kHz}$ as a function of temperature between 50 and $90^{\circ} \mathrm{C}$ in $5^{\circ} \mathrm{C}$ increments. The temperature was ramped down from $90^{\circ} \mathrm{C}$ to 
$50^{\circ} \mathrm{C}$ and then back up in quick succession to investigate sensor hysteresis.

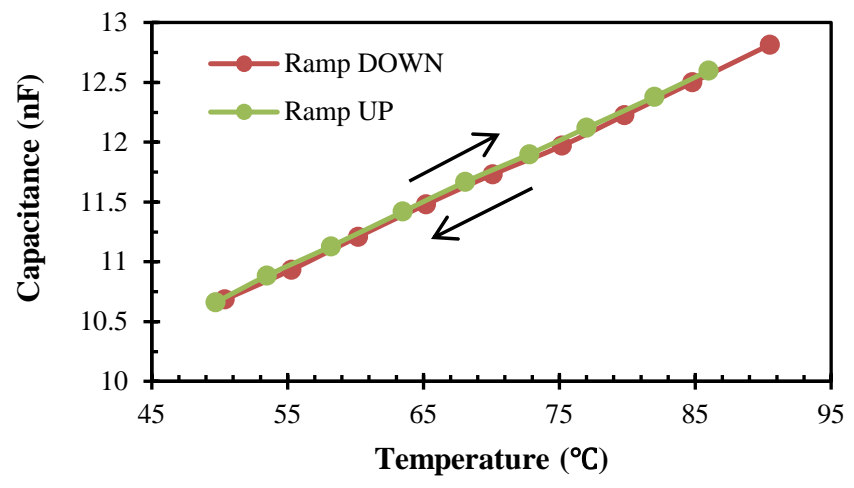

Fig. 3. Capacitance of ferroelectric PZT sensor as a function of temperature. Temperature was ramped down the up.

Fig. 3 also shows that PZT capacitance increases linearly as a function of sensor temperature between 50 and $90^{\circ} \mathrm{C}$, displaying a capacitance sensitivity of $52.8 \mathrm{pF} /{ }^{\circ} \mathrm{C}$. The graph also confirms that the PZT sensor displays no hysteresis over the $40^{\circ} \mathrm{C}$ temperature range explored.

However, for applications more suited to use on the human body, the sensor must be capable of detecting temperature through changes in ferroelectric layer capacitance closer to the human body temperature range $\left(\sim 37^{\circ} \mathrm{C}\right)$. Therefore, Fig. 4 presents the effect of PZT sensor temperature between room temperature $\left(\sim 22^{\circ} \mathrm{C}\right)$ and $45^{\circ} \mathrm{C}$ in much smaller steps. Due to the lack of hysteresis shown in Fig.3, for this experiment, temperature was only ramped in one direction (down).

Fig. 4 shows that PZT sensor capacitance increases linearly as a function of temperature between room temperature and $45^{\circ} \mathrm{C}$. This is in agreement with results shown previously in Fig.3 for higher temperatures. The data in Fig. 4 shows a sensitivity of $54.7 \mathrm{pF} /{ }^{\circ} \mathrm{C}$.

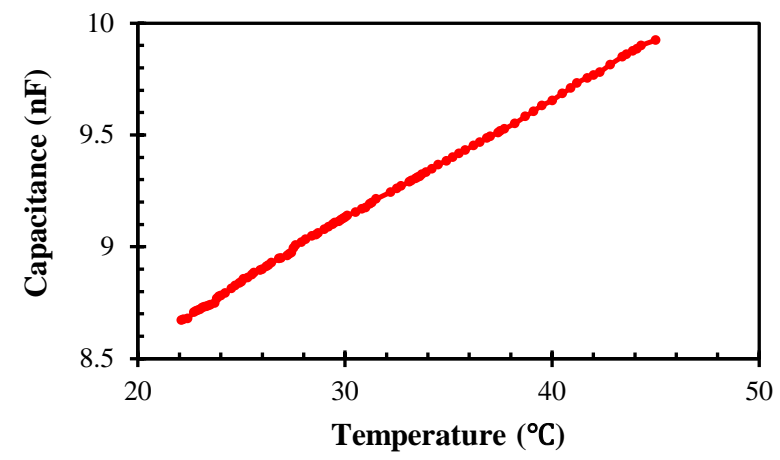

Fig. 4. Capacitance of ferroelectric PZT sensor as a function of temperature between 45 and $\sim 22{ }^{\circ} \mathrm{C}$ in $0.5^{\circ} \mathrm{C}$ increments.

\section{B. Response of PZT to Temperature Using Arduino Uno}

Using the Arduino Uno platform described in Section II, the PZT sensor was connected to the Uno to monitor sensor capacitance continuously in real-time and provide an average value every 5 seconds. Due to the effect of the capacitor connected in parallel with the sensor at the input to reduce the pyroelectric charge effects, the actual capacitance measured by the Arduino is a combination of the two capacitors in parallel. Therefore, the effect of the additional capacitor is subtracted in the Arduino code to provide the PZT sensor capacitance only. Fig. 5 shows PZT sensor capacitance between 36 to $50^{\circ} \mathrm{C}$ as monitored by the Uno platform. We have tried to remove the pyroelectric charge effects of PZT sensor by heating up this sensor above $400{ }^{\circ} \mathrm{C}$. This attempt was not successful and moreover, this caused the severed damage to the sensor. Therefore, we have to use a "fresh" PZT sensor with Arduino Uno measurement, i.e. a different sensor to that used previously in Figs. 3 and 4. The measurement in the temperatures between 0 and $90{ }^{\circ} \mathrm{C}$ has no effect for the measurement reproducibility.

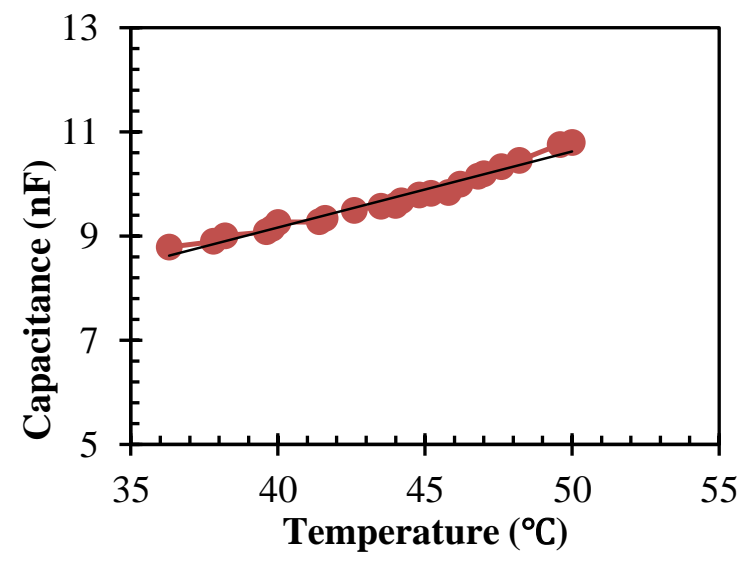

Fig.5 Ferroelectric PZT sensor capacitance measured using an Arduino Uno as a function of temperature between 36 and $50^{\circ} \mathrm{C}$.

Referring to the graph in Fig. 5, the sensor capacitance measured via the Uno displays an approximate linear dependence on PZT temperature between 36 and $50^{\circ} \mathrm{C}$. In this instance, using a slightly different sensor to before when the LCR meter was used, the capacitance sensitivity is $~ 146$ $\mathrm{pF} /{ }^{\circ} \mathrm{C}$.

\section{DISCUSSION}

In all cases explored, the capacitance of the PZT ferroelectric sensor displays a linear dependence on temperature. PZT temperature was varied between room temperature $\left(\sim 22^{\circ} \mathrm{C}\right)$ and $90^{\circ} \mathrm{C}$. Using the $\mathrm{LCR}$ meter to measure PZT capacitance, a sensitivity of $52.8 \mathrm{pF} /{ }^{\circ} \mathrm{C}$ was obtained between 50 and $90^{\circ} \mathrm{C}$, and a sensitivity of $54.7 \mathrm{pF} /{ }^{\circ} \mathrm{C}$ between $\sim 22$ and $45^{\circ} \mathrm{C}$. In addition, no temperature hysteresis was observed. This enables the exploitation of changes in PZT capacitance for temperature monitoring.

As a first step towards developing a wearable electronics platform for temperature monitoring using a ferroelectric capacitor, the PZT sensor was attached to an Arduino Uno platform. The Uno enabled the continuous measurement of the capacitance in real-time and provided an average capacitance of 10 readings every 5 seconds. This concept could be extended to design a small, lightweight PCB that could be attached to a "smart-shirt". Previously, a PCB has been used to provide the electronics for a wearable ECG sensor comprising conductive threads. An embroidery machine (Barudan BESTS1501) was used to create the electrically conductive paths and ECG electrodes [11]. Skin temperature for various body 
locations and different ambient temperatures was presented in [12] and this information is show in Fig. 6. The sensor position in smart shirt should be stable, comfortable for the user and should not be exposed to the trench. Therefore, Upper Back (Fig, 6, point D) was chosen for the sensor position.

\begin{tabular}{|c|c|c|c|c|}
\hline & Skin Location & Cold $\left(15^{\circ} \mathrm{C}\right)$ & Room $\left(27^{\circ} \mathrm{C}\right)$ & Hot $\left(47^{\circ} \mathrm{C}\right)$ \\
\hline & Forehead (A) & 31.7 & 35.2 & 37 \\
\hline & Back of Neck (B) & 31.2 & 35.1 & 36.1 \\
\hline & Chest (C) & 30.1 & 34.4 & 35.8 \\
\hline & Upper Back (D) & 30.7 & 34.4 & 36.3 \\
\hline & Lower Back (E) & 29.2 & 33.7 & 36.6 \\
\hline & Upper Abdomen (F) & 29.0 & 33.8 & 35.7 \\
\hline $\mathrm{m}$ & Lower Abdomen (G) & 29.2 & 34.8 & 36.2 \\
\hline & Tricep $(H)$ & 28.0 & 33.2 & 36.6 \\
\hline 0 & Forearm (J) & 26.9 & 34.0 & 37.0 \\
\hline & Hand (L) & 23.7 & 33.8 & 36.7 \\
\hline & Hip (M) & 26.5 & 32.2 & 36.8 \\
\hline & Side thigh (N) & 27.3 & 33.0 & 36.5 \\
\hline & Front Thigh (O) & 29.4 & 33.7 & 36.7 \\
\hline & Back Thigh (P) & 25.5 & 32.2 & 36.0 \\
\hline & Calf $(Q)$ & 25.1 & 31.6 & 35.9 \\
\hline$\Sigma$ & Foot (R) & 23.2 & 30.4 & 36.2 \\
\hline
\end{tabular}

Fig. 6. Skin temperature for various body location.

Ferroelectric sensors are suitable candidates for monitoring temperature for a range of applications. However, in their ferroelectric state, i.e. when all the dipoles in the unit cells are aligned, they display remarkable piezo- and pyroelectric properties upon application of dynamic pressure or temperature.

Currently, the ferroelectric PZT sensor itself is rigid in nature, however, ferroelectric polymer materials such as polyvinylidene fluoride (PVDF) or its copolymer poly(vinylidenefluoride-co-trifluoroethylene) (P(VDF-TrFE)) are capable of being processed onto flexile substrates, thus enabling the full integration of such sensors with a textilebased platform. In their poled/ferroelectric state these polymers display excellent piezo- and pyroelectric properties upon application of dynamic pressure or temperature. These polymers also exhibit a strong change in their capacitance with temperature that occurs both in the ferroelectric or paraelectric state. Previously, the capacitance of a P(VDF-TrFE) ferroelectric sensor showed an increase in its capacitance of $\sim 7$ $\mathrm{pF} /{ }^{\circ} \mathrm{C}[12]$.

\section{CONCLUSION}

This paper presents a cheap and robust platform capable of monitoring static temperature changes using a commercially available PZT ferroelectric sensor. The capacitive response of the PZT sensor has been examined for temperatures ranging from $\sim 22^{\circ} \mathrm{C}$ to $90^{\circ} \mathrm{C}$. Sensor capacitance shows a linear dependence as a function of temperature, with sensitivity in the range of $\sim 53 \mathrm{pF} /{ }^{\circ} \mathrm{C}$. To provide initial electronics capable of measuring PZT sensor capacitance continuously and in realtime, the PZT sensor was attached to an Arduino Uno and code was developed to measure capacitance as a function of sensor temperature. The sensor and Uno setup provides a proof of concept, which could be further developed using a combination of ferroelectric polymers and a light-weight PCB to create a completely wearable, low-cost, static temperature monitoring system for applications including human body temperature sensing.

\section{ACKNOWLEDGMENT}

This project received funding from the European Union's Horizon 2020 research and innovation program under the Marie Skłodowska-Curie grant agreement No 734331. $\mathrm{H}$. is a recipient of a doctoral training grant funded by the Engineering and Physical Sciences Research Council (EP/L505080/1). Funding also came from the Slovak Research and Development Agency under science fund grant No. APVV-16-0190: Research of integration of textile functional system for biodata monitoring aimed at achievement synergy of human health, comfort and safety and European Regional Development Fund under grant No. ITMS: 26220220022: Design of New Diagnostic Algorithm for Selected Tumour Affections.

\section{REFERENCES}

[1] J. Hayward, G. Chansin and H. Zervos, "Wearable technology 2016 2026. Markets, players and 10-year forecasts". IDTechEx Report, July 2016.

[2] L. Capineri, "Resistive Sensors with Smart Textiles for Wearable Technology: From Fabrication Processes to Integration with Electronics," in Eurosensors 2014, the XXVIII ed. of the conf. series, Brescia, Italy, 2014.

[3] J. Roh and S. Kim, "All-Fabric Intelligent Temperature Regulation System for Smart Clothing Applications," Journal of Intelligent Material Systems and Structures, vol. 27, pp. 1165 - 1175, 2016.

[4] Y. Lee and W. Chung, "Wireless Sensor Network Based Wearable Smart Shirt for Ubiquitous Health and Activity Monitoring," Sensors and Actuators B: Chemical, vol. 140, pp. 390 - 395, 2009.

[5] G. Schwartz, B. Tee, J. Mei, A. Appleton, D. Kim, H. Wang and Z. Bao, "Flexible polymer transistors with high pressure sensitivity for application in electronic skin and health monitoring," Nature Communications, vol. 4. p. 1859, 2013.

[6] S. Kim, J. Kim, Y. Kim, H. Song and C. Park, "Resistance Temperature Detector Sensor with a Copper Pattern on the Printed Circuit Board," Int. Journal of Control and Automation, vol. 8, pp. 67 - 74, 2015.

[7] S. Pullano, S. Islam and A. Fiorillo, "Pyroelectric Sensor for Temperature Monitoring of Biological Fluids in Microchannel Devices," IEEE Sensors Journal, vol. 14, pp. 2725 - 2730, 2014.

[8] J. Yun and S. Lee, "Human Movement Detection and Identification Using Pyroelectric Infrared Sensors," Sensors, vol. 14, pp. 8057 - 8081, 2014.

[9] H. Ma, Q. Huang, M. Qin and T. Lu, "A Micromachined Silicon Capacitive Temperature Sensor for Wide Temperature Range Applications," Journal of Micromechanics and Microengineering, vol. 20, p. 055036, 2010.

[10] "Murata Piezo Element Diaphragm", http://www.newark.com/murata/7bb-20-610/piezoelectric-diaphragms-63khz/dp/99K0276, accessed 19/04/17.

[11] R. Hudec, M. Benco, S. Matuska et. al., "Utilisation of electroconductive mixed Ag-yarns as data and power wires in an intelligent textile structures" in ELEKTRO 2014, Rajecke Teplice, Slovakia 2014

[12] F. Suarez, A. Nozariasbmarz, D. Vashaee et. Al., "Designing thermoelectric generators for self-powered wearable electronics", Energy \& Environmental Science, vol. 9, p. 2099-2113, 2016

[13] S. Hannah, S. Khan, D. Uttamchandani, R. Dahiya and H. Gleskova, "Response of P(VDF-TrFE) Sensor to Force and Temperature," in $11^{\text {th }}$ In.t Conf. on PhD Res. in Microelectronics and Electronics (PRIME), Glasgow, UK, 2015. 